\title{
Modern concepts of the fibrinolytic system
}

\author{
Sergei Sorokin ${ }^{1, *}$, Mark Shamtsyan ${ }^{1}$, and Nicolai Petrishchev ${ }^{2}$ \\ ${ }^{1}$ Saint-Petersburg State Institute of Technology, 198013, 26, Moskovskii pr., St. Petersburg, Russia \\ ${ }^{2}$ Pavlov First Saint Petersburg State Medical University, 197022, 6-86 L'va Tolstogo st., St. \\ Petersburg, Russia
}

\begin{abstract}
Among all medical pathologies, cardiovascular diseases are the most dangerous and deadly. On the one hand, thrombosis is a natural reaction of a person to damage to blood vessels, on the other hand, thrombosis is often the cause of death in the event of cardiovascular disease. There is a natural process called fibrinolysis to dissolve a blood clot after the bleeding has stopped and preventing vascular occlusion. This article examines current understanding of the human fibrinolytic system, main elements of fibrinolytic system and also addresses the topic of thrombolytic therapy.
\end{abstract}

\section{Introduction}

The formation of fibrin and its breakdown are fundamental processes of tissue repair in the human body. Any damage to the vascular bed must be blocked and subsequently repaired to ensure vascular integrity. Vascular damage leads to the activation of platelets and coagulation factors. Subsequent interactions between subendothelial material, endothelial cells, platelets, and proteins of blood coagulation and fibrinolysis are responsible for the formation and degradation of fibrin. In a healthy human body, these systems are believed to be in a delicate balance, that is, in a hemostatic, ensuring the integrity of the vascular system, and acute disturbances of this balance can lead to thrombosis or bleeding, while long-term and more moderate imbalance can impair tissue remodeling and repair [1].

Cardiovascular diseases are the most common cause of death worldwide, claiming about 17 million lives annually [2]. According to the statistics of the medical and prophylactic institution of the Administrative Department of the President of the Russian Federation, in the first 3 hours from the onset of an anginal attack, only $25 \%$ of patients are admitted to hospitals, and during the first 6 hours $-50 \%$ of patients. Drug therapy with the use of thrombolytics allows to achieve early reperfusion, complete restoration of blood flow in the infarct-associated artery is possible in $60 \%$ of patients [3]. Open surgeries in neurology and neurosurgery are increasingly being replaced by minimally invasive interventions, which allow, while maintaining the radicality of hemorrhage removal, to improve the functional outcomes of the disease. One of the promising areas of minimally invasive surgery for hemorrhagic stroke is the method of local fibrinolysis of hemorrhages [4, 5].

* Corresponding author: sersorock@yandex.ru 


\section{Fibrinolytic system}

The fibrinolytic system is an integral part of the hemostatic system, since it always accompanies blood coagulation and is even activated by the same factors as the hemocoagulation process [4].

Plasma elements, platelets and other cells are involved in the process of fibrinolysis. Fibrinolysis can occur:

- either under the direct influence of enzymes that destroy fibrin threads (fibrinolysin),

- either indirectly, through the activation of an inert plasma protein, plasminogen.

The main enzyme that breaks down fibrin is plasmin, which is formed from inactive plasminogen during activation. The process of plasminogen activation includes 3 pathways:

- internal;

- external;

- exogenous [6].

The external path is the main one, however, both the internal and exogenous paths play an important role. A feature of the internal pathway of fibrinolysis is the simultaneous activation of its products, namely the factor Xia, the internal pathway of coagulation. The internal pathway of fibrinolysis accounts for about $15 \%$ of all fibrinolytic activity. Activation of plasminogen via the intrinsic pathway occurs with the participation of factor XII, prekallikrein, high molecular weight kininogen and factor XI [7].

Prekallikrein and factor XI are able to bind to cytokeratin, which is a kininogenic binding receptor on the surface of endothelial cells, platelets and granulocytes. The relationship of factor XI and prekallikrein with cytokeratin via kininogen depends on the presence of $\mathrm{Zn}^{2+}$. The combination of prekallikrein and kininogen on endothelial cells triggers the activation of membrane-bound cysteine protease, which in turn activates prekallikrein to kallikrein. Kallikrein then breaks down kininogen, resulting in the formation of bradykinin and increased binding of kininogen to endothelial cells. The formation of bradykinin inhibits the activation of thrombin-induced platelets, and bradykinin is also involved in fibrinolysis, since it stimulates vascular endothelial cells to release tissue plasminogen activator. The resulting kallikrein is a potent activator of factor XII, converting it to factor XIIa, which subsequently converts factor XI to factor XIa [7].

The subsequent activation of plasminogen is directly induced by factor XIIa, kallikrein and factor Xia. The researchers also associate the main part of plasminogen activation via the intrinsic pathway with a plasminogen proactivator (factor XII-PA) [8]. The activation of this activator depends on the presence of factor XII, prokallikrein, and kininogen $(120,000)$. Attempts have been made to isolate and characterize factor XII-PA; however, it is unclear whether it exists as a specific plasma protein. Recent studies show that factor XIIdependent plasminogen is activated by $30,000 \mathrm{~g} / \mathrm{mol}$ of protein. The activity of the plasminogen activator of this protein probably depends on the presence of a cofactor [7,8].

The external pathway of plasminogen activation occurs with the participation of two main activators: tissue (TPA) and urokinase (UPA) types [9]. This transformation occurs predominantly on the surface of fibrin fibers, after which the resulting plasmin cleaves fibrin. The attachment of plasminogen and TPA to the fibrin clot is mediated by free Cterminal lysine residues on partially cleaved fibrin, as well as by specific lysine-binding sites in plasminogen and TPA molecules. Most of the active plasmin is formed on fibrin after the formation of the fibrin / TPA / plasminogen triple complex. Since plasmin cleaves peptide bonds formed by lysine residues in fibrin, new $\mathrm{C}$-terminal lysine residues are formed in the process of fibrinolysis, which serve to bind additional plasminogen and TPA molecules, thereby accelerating and enhancing the process of plasmin formation and cleavage of fibrin by the positive feedback mechanism ... As a result, the entire polymeric 
fibrin network breaks down to soluble fibrin fragments, which are removed from the bloodstream [9].

The exogenous pathway of fibrinolysis activation is associated with bacterial proteins, in particular streptokinase and staphylokinase. At the first stage of fibrinolysis, the Xfragment is detached, which is then cleaved into Y- and D-fragments [10]. The X-and Yfragments are called "early", or high-molecular, fibrin and fibrinogen degradation products. The Y-fragment is further degraded into an E-fragment and another D-fragment. D- and Efragments are "late", or low-molecular. As a result of complete degradation of the fibrin clot, D-dimers (D-D) are formed [10,11].

A high level of D-dimers is a recognized laboratory marker of thrombinemia and intravascular coagulation in pathological conditions such as disseminated intravascular coagulation syndrome (DIC), deep vein thrombosis of the lower extremities and pulmonary embolism. The concentration of D-dimers is taken into account in the diagnosis of acute aortic dissection $[10,11]$.

\section{Elements of the fibrinolytic system}

\subsection{Fibrinogen and fibrin}

Fibrinogen, a precursor of fibrin, circulates in the blood at a concentration of $8.8 \mu \mathrm{M}$; its molecular weight is $340 \mathrm{kDa}$. Unlike most plasma proteins, fibrinogen has a pronounced non-globular shape, resembling an ellipsoid $45 \mathrm{~nm}$ long and 5-9 nm thick. Fibrinogen is a dimer, each half of it consists of three polypeptide chains linked by disulfide bridges. Structurally, the fibrinogen molecule is presented in the form of several domains: two Ddomains, a central E-domain, and two $\alpha \mathrm{C}$ domains. The halves of the dimer are linked to each other at the $\mathrm{N}$-termini of all three pairs of polypeptide chains (E-domain), where the active sites of the molecule are located. Fibrinogen is activated by cutting off fibrinopeptides A and B with thrombin. First, fibrinopeptide A is cleaved - this is enough to start polymerization, fibrin molecules begin to form a polymer thread. After cleavage of fibrinopeptides B, the fibrin strands are allowed to aggregate laterally, forming a threedimensional fibrin network. Additionally, the fibrin filaments are strengthened by crosslinking due to factor XIIIa (in addition, factor XIIIa attaches a2-antiplasmin molecules to the fibrin filaments, which impairs the lysis process). The parameters of the fibrin network (fibril thickness and average length between branches) depend on the polymerization conditions - $\mathrm{pH}$ of the medium, salt concentration, ionic strength, thrombin concentration $[6,12]$.

\subsection{D-dimer}

The product of fibrin degradation, indirectly reflecting the result of the joint work of the coagulation system and fibrinolysis. Low concentration of D-dimer corresponds to the normal state of the hemostatic system; an increase in D-dimer is observed in thrombosis, pregnancy, in the elderly and in a wide range of inflammatory and oncological conditions [11]. In this regard, the specificity of such a test for assessing the state of fibrinolysis is rather low. Usually this method is used to exclude deep vein thrombosis and PE, assuming normal functioning of the fibrinolytic system. In addition, this test shows a false positive result in lipemia, hyperbilirubinemia, hemolysis, and an increased level of rheumatoid factors. Immunological methods for measuring D-dimer show high sensitivity against thrombosis (93-96\%), but low specificity (43-53\%). D-dimer test readings are 
recommended for use in scoring calculations of the probability of deep vein thrombosis of the lower extremities and pulmonary embolism [6].

\subsection{Plasminogen and plasmin}

The main protein of the fibrinolysis system is plasmin. It is on it that most of the feedback loops are closed, it is he who conducts the only physiologically significant reaction of fibrinolysis - the hydrolysis of fibrin filaments. Plasmin, like most coagulation and lysis enzymes, is a serine proteinase. It is formed from its precursor, plasminogen (Pg), which circulates in the blood at a concentration of $2 \mu \mathrm{M}$. Plasminogen is a single chain glycoprotein with a molecular weight of $92 \mathrm{kDa}$. The lifetime of free plasminogen is about two days. The main organ that synthesizes plasminogen is the liver, but this protein is present in the extravascular space of many other tissues. It is known that in addition to the liver, it can be synthesized by the kidneys and the cornea [6].

The parent plasminogen is called Glu-Plg because it contains a glutamic acid residue at the N-terminus. The Glu-Plg molecule consists of three parts: an N-terminal "preactivation" peptide, five homologous domains called kringles (K1 - K5), and a catalytic domain that has its own proteolytic activity. Kringles (named for their structural similarity to the Scandinavian pretzel - kringle) provide binding of plasminogen to fibrin, as well as to cofactors and cellular receptors. The transformation of inactive plasminogen into proteolytically active plasmin consists in cleavage of the Arg561-Val562 peptide bond, catalyzed by plasminogen activators. Simultaneously, the newly formed plasmin cleaves several peptide bonds in the parent plasminogen, which leads to the formation of a truncated form of plasminogen, designated Lys-Plg, with a lysine residue at the N-terminus of the molecule. The conversion of Glu-Plg to Lys-Plg is another positive feedback mechanism, since Lys-Plg binds better to fibrin and is converted to plasmin faster than GluPlg. The transition of the plasmin molecule into a double-stranded form is also noted (the chains are connected by two disulfide bridges). Plasminogen mutations can cause pseudomembrane diseases such as fibrous (lignus) conjunctivitis. Plasminogen-deficient mice also exhibit fibrous conjunctivitis, thrombosis, and fibrin deposits in the intra- and extravascular space of many tissues. The concentration of plasminogen in human blood plasma and its activity are measured mainly using a functional chromogenic test. Streptokinase is used as an external plasminogen activator, which forms an autocatalytic complex with it, initiating the formation of plasmin, which in turn activates a chromogenic tag. A decrease in the functional activity of plasminogen is observed in some ethnic groups (mainly in Asian countries), but no association with an increased risk of thrombosis has been identified. Even in the case of homozygous plasminogen deficiency, thrombotic pathologies are not observed in such patients. The absence of thrombotic pathologies in such patients is probably due to the fact that even low concentrations of plasminogen (from 4 to $51 \%$ ) are sufficient for the normal functioning of the fibrinolysis system [6].

\subsection{Plasminogen activators}

There are two main plasminogen activators in our body - urokinase plasminogen activator (UPA) and tissue plasminogen activator (TPA). Plasminogen activators are used in patients in the treatment of thrombosis and thrombotic complications. In addition to physiological plasminogen activators, the clinic uses animal and bacterial activators. The most common of these are streptokinase [6] and staphylokinase [13]. With streptokinase, plasmin forms an autocatalytic complex, which transfers other plasminogen molecules into an active state [6]. TPA and staphylokinase bind to fibrin and thus avoid rapid inactivation, whereas UPA and 
streptokinase do not bind to fibrin and can activate plasminogen both in the circulating blood and on the surface of the clot, but are quickly inactivated themselves [13].

\subsubsection{Tissue plasminogen activator (TPA)}

TPA is a $68 \mathrm{kDa}$ serine proteinase. TPA is secreted mainly by endothelial cells; therefore, it is also called a vascular plasminogen activator. In plasma, TPA circulates at a concentration of $70 \mathrm{pM}$, and only $20 \%$ of this amount is in free form, the rest of TPA molecules are in a bound state with their inhibitor (PAI) [6].

TPA is secreted as a single-chain form (o-TPA). When interacting with plasmin, which cleaves the Arg275-Ile276 peptide bond, TPA transforms into a double-stranded form (dTPA). An interesting fact is that o-TPA, although it is an enzyme, in the absence of fibrin exhibits very low activity towards plasmin, although it is capable of activating plasminogen. With the appearance of fibrin, the formation of triple complexes (TPAPgfibrin) begins, and the activity of o-TPA increases approximately 1000 times [6].

Experimental data on the kinetics of plasminogen activation with TPA vary greatly, apparently, this is due to the fact that plasminogen activation is influenced by many factors: various procedures for the preparation of TPA and plasminogen, different concentrations of substrates, and in the study with fibrinogen and its derivatives - more and conditions for the formation of fibrin [6].

Since TPA has a high specificity for plasmin and begins to work directly in the area of a thrombus (for this, it needs fibrin), it became the main target for researchers in the field of genetic engineering - they aimed to improve its performance, trying to create an effective therapeutic drug. The most successful in this direction was the creation of a series of TPA mutants resistant to PAI-1 (its main inhibitor). A group of researchers found an interaction between a positively charged site of TPA (298-302) and a negatively charged site of PAI-1 (350-355). Mutagenesis in residues Arg298, 299, and 304 of the TPA molecule made it possible to create a mutant molecule that is 120,000 times worse inhibited than ordinary TPA molecules. These studies formed the basis for the creation of Tenecteplase, a TPA mutant, in which the sequence 296-299 (Lys-His-Arg-Arg) was replaced by four alanines. This mutant showed a slower clearance from the bloodstream and a higher selectivity for fibrin [6].

The expression of TPA is influenced by such vasoactive substances as thrombin and histamine; they interact with cells through G-protein receptors. Note that not all endothelial cells synthesize TPA; It is known that TPA expression is limited in small vessels. Free TPA and complexes of TPA with inhibitors are rapidly removed from the bloodstream by binding to receptors of endothelial cells and hepatocytes. The elimination half-life is normally about 3 minutes [6].

Cases of congenital deficiency of TPA and UPA in humans have not been described. Deficiency of these proteins was believed to be incompatible with life. However, experiments on mice showed an unexpected result: mice deficient in TPA developed normally. Microscopic analysis revealed mild glomerulonephritis in these mice. Lysis of the clot was worse for them than for wild-type individuals. Complete knockout of activators is similar in phenotype to knockout of plasminogen itself; this shows that TPA and UPA are the main plasminogen activators in mammals [6].

When measuring the level of TPA, as a rule, an inactive complex with PAI-1 is recorded; therefore, an increased level of such TPA can characterize not an increase in the activity of fibrinolysis, but, on the contrary, its inhibition. In some laboratories, a fibrinolytic test of venous occlusion using a cuff is performed: a cuff is put on the patient's arm to measure pressure; for 10-20 minutes, pressure is injected - an average between systolic and diastolic (but $<100 \mathrm{~mm} \mathrm{Hg}$ ). Blood is drawn before and after the procedure, 
below the cuff, which is still inflated. It is believed that the result of this procedure is the release of endothelial lysis factors, but it is not clear whether this procedure reflects the physiological state of the patient better than the usual blood collection [6].

It can be assumed that such an unclear picture describing the relationship between the level of TPA and clinical manifestations of hemostasis / fibrinolysis disorders is associated with the fact that TPA is an acute phase protein: at rest, trace concentrations of TPA are present in the blood, and most of it leaves the endothelium under the action of thrombin formed during blood coagulation. The usual analysis of venous blood will not show how much TPA is released from the endothelium and, accordingly, how the clot lysis will proceed [6].

\subsubsection{Urokinase plasminogen activator (UPA)}

UPA is detected in urine at a concentration of $40-80 \mu \mathrm{g} / \mathrm{L}$. It is synthesized by a number of cells - epithelial, connective tissue, macrophages, endothelial cells, etc. UPA activates plasminogen by cleaving the Arg561 - Val562 bond, in the same way as TPA, but unlike the last UPA, this reaction does not require the presence of fibrin. This property suggests that the main physiological role of UPA is participation in the processes of destruction of the extracellular matrix, cell migration, inflammation, wound healing, metastasis, while the main task of TPA is the destruction of fibrin clots in the blood [6].

UPA is synthesized in the form of a single-stranded form, which under the action of plasmin transforms into a double-stranded form, which increases its activity in the reaction of plasminogen to plasmin conversion by 250 times. The half-life of UPA from plasma is about $7 \mathrm{~min}$, cleavage occurs in the liver. Upon binding to the urokinase receptor (UPA-R), the activity of o-UPA increases by two orders of magnitude. It is assumed that due to this, monocytes can migrate into the thrombus [6].

No genetic diseases associated with the deficiency of UPA and UPAR have been recorded. Studies on mice with knockout of the UPA gene have shown that such mice (UPA - / -), like TPA - / - mice, have fibrin deposits in their organs, however, they do not suffer from spontaneous thrombus formation. In UPA - / - mice, the wound healing process is significantly slowed down. Interestingly, in mice with UPAR - / - wounds heal in the same way as in healthy individuals [6].

\subsection{Fibrinolysis inhibitors (plasmin inhibitors, PAI, TAFI)}

By their mechanism of action, inhibitors of the fibrinolysis system are divided into several types. The first group (PAI-1, PAI-2) inhibits plasminogen activators, urokinase and tissue activator. Proteins of the second group (antiplasmins and macroglobulins) directly inhibit plasmin. The third group includes TAFI, the inhibitory effect of which is associated with the removal of plasmin and TPA binding sites on fibrin molecules [6].

\subsubsection{Plasminogen activator inhibitors (PAI)}

PAI-1 is the main inhibitor of plasminogen activators, urokinase and tissue activator, belongs to the superfamily of proteins serpin (serine protease inhibitor) [14]. The PAI molecule has the property of autoinactivation (occurs due to the spontaneous movement of the active center loop into the interior of the molecule). This autoinactivation is passive and reversible (activity can be restored using denaturation). The half-life of PAI-1 is short about 10 minutes; plasma concentration varies widely - from 1 to $40 \mathrm{ng} / \mathrm{ml}$. PAI-1 is synthesized by many tissues. The main contribution to the synthesis of PAI- 1 in the body is made by platelets, endothelial cells, hepatocytes and adipocytes. About half of the amount 
of circulating PAI is contained in platelets, which release it during the formation of a thrombus, as a result of which the thrombus acquires resistance to lysis [6].

PAI-1 deficiency is rare in humans and is usually accompanied by prolonged and delayed bleeding. Oral administration of tranexamic acid normalizes the state of hemostasis in these patients. An increased level of PAI-1 is associated with distinguishable CVS pathologies, oncology, obesity, type 2 diabetes mellitus, etc. [6]

PAI-2 is serpin [14], first found in the placenta and therefore considered a placental UPA inhibitor. PAI-2 plays the role of an inhibitor of plasminogen activators in the tissues of the epidermis, esophagus, cornea, tongue, and vagina. Normally, PAI-2 is not detected in plasma, appearing only during pregnancy. The concentration of PAI-2 reaches its maximum at the $33 \mathrm{rd}$ week of gestation - about $250 \mathrm{ng} / \mathrm{ml}$. PAI-2 is also found in patients with myeloid leukemia types M4 and M5, in patients with sepsis - they also have an increased level of PAI-1 [6].

Measurement of the concentration of PAI-1 in plasma does not reflect its real inhibitory ability, since most of PAI-1 is released by activated platelets directly in the thrombus [6]

The PAI-1 level may increase in diabetes mellitus and insulin resistance, during pregnancy, and changes depending on the circadian rhythm. Studies of the dependence of the risk of thrombotic diseases on the level of PAI-1 reveal the same heterogeneous picture as for HAT, showing either positive, then zero, or negative correlation. Apparently, this is also due to the fact that PAI-1 is released from platelets during thrombus formation, and locally its concentration may increase several times compared to the baseline level (during blood sampling) [6].

\subsection{2 a2-Antiplasmin}

a2-Antiplasmin, or $\alpha 2$-plasmin inhibitor, $63 \mathrm{kDa}$ serpin, plays the role of the main plasmin inhibitor in the body. The concentration of $\alpha 2$-antiplasmin in plasma is $70 \mathrm{mg} / \mathrm{L}(1 \mu \mathrm{M})$, which corresponds to approximately half the concentration of plasminogen in molar equivalent. The half-life of the free molecule is three days (the antiplasmin-plasmin complex is excreted in 12 hours). Antiplasmin, like plasmin, can bind to fibrin via lysinebinding sites. Plasmin, bound in this way to fibrin, becomes protected from inactivation by $\alpha 2$-antiplasmin [6].

An increased level of plasmin-antiplasmin complexes, a decrease in the concentration of antiplasmin characterizes a hyperfibrinolytic state, which can be caused by trauma, disseminated intravascular coagulation, and acute promyelocytic leukemia. However, no correlation was established between the concentration of antiplasmin and the severity of pathologies, apparently due to the high baseline level of antiplasmin. Even with a deviation of several times from the baseline value, the concentration of antiplasmin remains high enough to maintain relatively normal lysis under normal conditions and does not lead to a change in the clinical picture. The antiplasmin test is used to diagnose rare genetic diseases that can affect the outcome of major surgery when hemostasis functions outside of normal conditions. [6]

\subsubsection{Lipoprotein (a)}

Lipoprotein (a) is a plasma lipoprotein similar in structure to low density lipoprotein in that it consists of an apolar lipid core enclosed in a predominantly phospholipid monolayer with associated glycoproteins. Apoglycoprotein has strong structural homology with plasminogen and exists in several isoforms containing from 12 to 51 plasminogen 4-like units, as well as a plasminogen 5-like unit and an inactive protease region homologous to plasminogen. Due to the structural homology of the kringles, lipoprotein (a) can interfere 
with the binding of plasminogen to the lysine composition in fibrin and cell receptors and, thereby, disrupt fibrinolysis and pericellular proteolysis [14].

\subsection{4 a2-Macroglobulin}

Macroglobulin is a high molecular weight protein with a molecular weight of approximately $725 \mathrm{kDa}$, the main inhibitor of a broad spectrum of proteinases; it also plays the role of a reserve inhibitor for a number of enzymes, including plasmin. Its concentration in blood plasma is $3 \mu \mathrm{M}(2.5 \mathrm{mg} / \mathrm{ml})$. Macroglobulin begins to exert a noticeable effect on the fibrinolysis system when the reserves of $\alpha 2$-antiplasmin are depleted and the role of the main plasmin inhibitor is transferred to macroglobulin, although its efficiency with respect to plasmin is 10 times lower than that of $\alpha 2$-antiplasmin. Macroglobulin plays the role of a trap, capturing the target, as a result of which access to the substrate from the outside is blocked. [6] However, cell and fibrin bound plasmin is protected against $\alpha 2-\mathrm{MG}$ [14].

An increase in the concentration of macroglobulin in the blood is observed in diseases associated with liver fibrosis (cirrhosis, hepatitis, Wilson-Konovalov disease), nephrotic syndrome, prostate adenoma and diabetes mellitus. [6]

\subsubsection{Thrombin-activated fibrinolysis inhibitor}

TAFI is a single-chain glycoprotein with a molecular weight of $60 \mathrm{kDa}$. The concentration of TAFI in the blood can vary from tens to hundreds of $\mathrm{nM}$. Under the influence of the thrombin-thrombomodulin complex, TAFI is converted into an active form (TAFI). TAFI removes the $\mathrm{C}$-terminal lysine amino acid residues from fibrin, which prevents the binding of plasmin to the fibrin network. TAFI is unstable at $37^{\circ} \mathrm{C}$; the half-life of the molecule is 8-9 $\min [6]$.

A number of studies show a correlation between an increased level of TAFI and the risk of thrombosis, as well as an association with the level of proteins in the acute phase of inflammation [6].

TAFI is measured using both immunological and functional tests. Functional tests have the advantage of measuring only active TAFI, while immunological tests measure both active and inactivated TAFI. There are various ways of setting the TAFI functional test. The first method: the sample is diluted 20 times in TAFI-deficient plasma, thrombin is added to the solution, which activates clotting and TAFI itself, and recombinant TPA, which triggers lysis. The delay in lysis is then measured as compared to the control experiment. The second method: the test sample is activated with thrombin, after which the reaction is stopped by adding the ppack reagent (D-phenylalanyl-prolyl-arginyl chloromethyl ketone - low molecular weight thrombin inhibitor); the activity of the formed TAFI is measured using a substrate specific to it [6].

An increase in the TAFI level correlates with the likelihood of venous thromboembolism: a TAFI concentration above the 90th percentile corresponds to a twofold increase in the risk of DV. In addition, there is a known connection between TAFI and various pathological conditions: renal and hepatic failure, endocrine diseases, oncology, DIC, and pregnancy [6].

\subsection{Surface "fibrinolytic" cell receptors}

The plasminogen activation system appears to be widely involved in many physico-logical and pathological processes, in addition to fibrinolysis, through the generation of plasmin on the cell surface. Therefore, for plasminogen and its activators, there are many binding sites for cells or receptor molecules [14]. 


\subsubsection{Plasminogen receptors}

Plasminogen binding to cells is mediated by a heterogeneous population of plasminogen receptors. The interaction of plasminogen with the binding sites of the cell with the surface accelerates the conversion of plasminogen into plasmin, enhances the catalytic activity of plasmin itself, and protects bound plasmin from inactivation by inhibitors. Plasminogen receptors are present on platelets, monocytes, macrophages, neutrophils, endothelial cells, and some non-vascular cells [14].

\subsubsection{Receptor UPA and TPA}

The 55-kDa UPA receptor ( $\mathrm{u}-\mathrm{PAR}$ ) is required for the activation of plasminogen on the cell surface, mediated by strong binding of its specific ligand, UPA. The binding leads to a strong increase in the activation of plasminogen on the cell surface, the effect depends on the simultaneous binding of pro-UPA with u-PAR and plasminogen with its receptors on the cell surface. In addition to binding to u-PAR, UPA can cleave intact three-domain $u-$ PAR (I-III) molecules, releasing the ligand-binding domain I and leaving the cleaved form of u-PAR, uPAR (II-III), on the cell membrane. This cleavage inactivates the binding potential of u-PAR for UPA and vitronectin. PAI-1 can bind to u-PAR-linked UPA, inhibiting the degradation of extracellular matrix proteins, initiated by the binding of the cell to UPA. The inhibitory effect of PAI-1 can be eliminated either by UPA-induced cleavage and inactivation, or by the formation of a quaternary complex (u-PAR) - (UPA) (PAI-1) with $\alpha 2$-macroglobulin, which is then internalized and digested, while how u-PAR is recycled to the cell surface [14].

Binding of TPA together with plasminogen to cells can lead to accelerated generation of plasmin. Monocytes and monocytoid cells bind TPA and are able to stimulate TPA activity by about 20 times [14]. The role of TPA is mainly in fibrinolysis, and the role of UPA in pericellular proteolysis, cell migration, adhesion, invasion and other processes and functions associated with cells.

\subsection{Other modulators of fibrinolysis}

Several other inhibitors, such as PAI-3 (protein C inhibitor), neuroserpine, histidine-rich glycoprotein and protease nexin, have been shown to have little, local and / or uncertain effect on the activity of components of the human fibrinolytic system [14].

\subsubsection{Natural anticoagulants}

PDPs have a pronounced anticoagulant effect. They not only block fibrin, but also prevent the formation of prothrombin and polymerization of fibrin monomers, reduce or inhibit the adhesive and aggregation function of platelets. Determination of the nature and content of PDP is important in assessing the forms of DIC syndrome, since it determines the scale of intravascular coagulation [2].

Natural anticoagulants can be classified as primary and secondary. Primary ones are found in plasma and blood cells and act regardless of whether a blood clot is formed or dissolved. Secondary anticoagulants arise in the process of blood coagulation and fibrinolysis due to the proteolytic action of the enzyme on the substrate [2].

Antithrombin III (AT III) is the main physiological inhibitor of coagulation factors, it is able to block prothrombinase by both external and internal mechanisms, including factors XIIa, XIa, VIIIa, IXa, Xa, thrombin and kallikrein. AT III is synthesized in the liver and endothelium of the microvasculature. Heparin increases the degree of inhibition of AT III- 
coagulation factors by several thousand times. In addition to AT III, heparin cofactor II (HC II) [2] has inhibitory properties with respect to thrombin exclusively.

A potent physiological anticoagulant is the extrinsic coagulation pathway inhibitor (TFPI), or lipoprotein-associated coagulation inhibitor (LACI). TFPI is mainly synthesized in the endothelium of the microvascular bed, to a lesser extent by megakaryocytes and fibroblasts. TFPI is a cofactor for low molecular weight heparin (LMWH). LMWH is able to increase the level of TFPI in the blood by $500 \%$. TFPI is the most important inhibitor of complex VIIa - tissue factor (VIIa - TF). In addition, TFPI inhibits the Xa factor and to a lesser extent the IXa factor [2].

Other inhibitors of hemocoagulation are $\mathrm{C} 1$-esterase inhibitor, $\alpha 2$-macroglobulin, $\alpha 1$ antitrypsin [2].

Protein C (PC) is synthesized in the liver together with protein S (PS) and thrombomodulin (TM), it is an important regulator of the coagulation cascade, which functions according to the principle of negative feedback.

For the anticoagulant function of MS, its activation is required, leading to the formation of activated protein $\mathrm{C}$ (APC). This process is carried out with the participation of Xa-factor, thrombin, thrombomodulin (TM). The main value of APC is inactivation of $\mathrm{V}$, Va, and VIIa factors, which prevents the generation of the prothrombin activator complex. These reactions are enhanced in the presence of $\mathrm{Ca}^{2+}$ ions, anionic membranes and protein $\mathrm{S}$ (PS) (6) [2].

APC also enhances fibrinolysis, which is associated with its ability to neutralize the plasminogen activator inhibitor (PAI-1). The anti-inflammatory effect of APC is associated with inhibition of the production of pro-inflammatory cytokines, APC is inhibited by PAI1, $\alpha 1$-antiplasmin, $\alpha 2$-macroglobulin [2].

Thrombomodulin (TM) is localized on the surface of the endothelium, performs an anticoagulant function and provides thromboresistance of the vascular wall. TM has a positive effect on the processes of intrauterine development of the fetus. In the process of HM degradation, soluble HM appears in the bloodstream, which is regarded as a marker of endothelial damage and an early preclinical sign of preeclampsia [2].

In addition to primary natural anticoagulants, secondary anticoagulants are formed during blood coagulation. These include PDF, "waste", i.e. past activation phases, blood coagulation factors [2].

Pathological anticoagulants are absent in the blood under normal conditions, but appear in various immune disorders. These include antibodies to blood coagulation factors, most often to factors VIII and V (often occurring after childbirth and massive blood transfusions), and immune complexes - lupus anticoagulant [2].

\section{Thrombolytic therapy}

Therapeutic thrombolysis or thrombolytic therapy is the dissolution of blood clots by injecting exogenous Plg activators, such as those listed below, into the circulation and is a common treatment for acute myocardial infarction and stroke. Regardless of the drug, the method of its administration and the type of thrombolytic therapy, be it systemic or selective thrombolysis, the elimination of the occlusion and the dissolution of the thrombus should be ensured. A particularly important condition for the use of this therapy is the framework of the so-called "therapeutic window" - the time at which the treatment is most effective, and the disease itself passes with fewer consequences. For a disease such as ischemia, the "therapeutic window" is conventionally up to 6 ours, however, the formation of $50 \%$ of the heart attack occurs already in the first 1.5 hours after the onset of symptoms. In this regard, the restoration of blood flow - reperfusion - should be carried out in a shorter time frame, or the "therapeutic window" should be increased. For these purposes, 
thrombolytic therapy is used in emergency medicine, which allows reperfusion and, optimally, to dissolve the thrombus [14].

According to the recommendations, the use of systemic thrombolysis makes sense in the first 3 hours after the onset of stroke. Systemic thrombolysis involves the intravenous administration of a fibrinolytic drug - the drug is administered at a dosage of $0.9 \mathrm{mg}$ per 1 $\mathrm{kg}$ of body weight (maximum body weight $90 \mathrm{~kg}$ ), 10\% of the drug volume is injected bolus, followed by a 60 -minute intravenous infusion of the residual volume of the drug. It is possible to use thrombolytic therapy with a body weight of more than $90 \mathrm{~kg}$, but the effectiveness of thrombolytic therapy is significantly reduced, and in this case, we can talk about a negative correlation between indicators such as body mass index and the degree of therapy success. A known complication of systemic thrombolytic therapy is the risk of hemorrhagic hemorrhage [14].

In cases where the time from the onset of stroke is exceeded (more than 3 hours) or intravenous thrombolysis did not lead to the development of positive dynamics, selective (intra-arterial) thrombolysis is used [2]. This method is an invasive manipulation performed under X-ray control. A significant advantage of this technique is the application time up to 12 hours from the onset of the disease when the lesion is localized in the vertebrobasilar basin and up to 6 hours in other localization. Access to the occluded vessel is through the femoral artery, $1 \mathrm{mg}$ of a fibrinolytic - recombinant tissue plasminogen activator (rt-PA) is injected distal to the thrombus, $1 \mathrm{mg}$ directly into the thrombus, $1 \mathrm{mg}$ into the proximal thrombus border, followed by intra-arterial infusion of rt-PA at a dosage $17 \mathrm{mg}$ in 60 minutes. Selective thrombolytic therapy is characterized by greater clinical efficacy compared to intravenous rt-PA [14].

At a later date from the onset of stroke, intravascular thrombolytic therapy does not make sense, since its use outside the therapeutic window significantly increases the risk of reperfusion injuries and hemorrhagic complications, and therefore, after 6 hours, the only way to restore perfusion is thromboembolectomy, or "mechanical thrombolysis" [ five]. The application of this method is shown both in the case of ineffective selective thrombolysis, and within 8 hours from the onset of stroke [14].

Although all of the above recanalization techniques show different clinical efficacy in comparison, each has its own advantages and disadvantages. The main advantage of intravenous thrombolysis is that it is a non-invasive intervention, and the disadvantage is the lack of full control of the administration of a fibrinolytic agent and higher doses compared to selective thrombolysis, which increases the risk of hemorrhagic complications. The disadvantage of selective thrombolysis and thrombectomy is the invasiveness of both manipulations, as well as the fact that the procedure under X-ray control entails an additional radiation load on the body of both the patient and the medical staff. Also, as the disadvantages of the above methods, significantly higher material costs can be noted [14].

\subsection{Fibrinolytic drugs}

Fibrinolytics are classified into 3 groups:

1. fibrinolytics of a direct mechanism of action (plasmin);

2. fibrinolytics with an indirect mechanism of action - activators of profibrinolysin (alteplase, prourokinase, streptokinase, urokinase);

3. drugs that promote the release of tissue fibrinogen activator (nicotinic acid) [14].

Recombinant TPA, rt-PA, alteplase (Activase $\AA$, Actilyse ${ }^{\circledR}$ ) is a drug belonging to the family of serine proteases (tissue activator of fibrinogen), which is a fibrinolytic of an indirect mechanism of action of the 3rd generation, almost identical to wild-type TPA. With standard thrombolytic therapy, plasma concentrations of about $4000 \mathrm{ng} / \mathrm{ml}$ are achieved, which is almost 1000 times higher than physiological indicators. Alteplase is called a 
selective thrombolytic agent because it acts on plasminogen, which is closely related to fibrin. This drug has a moderate selective activity, it has no antigenic properties and the risk of developing allergic reactions is extremely low [14].

Reteplase (Retavase ${ }^{\circledR}$, Rapilysin ${ }^{\circledR}$ ) is a single-chain, non-glycosylated deletion mutant of rt-PA, consisting only of kringle 2 and a catalytic domain, which has plasminogenolytic activity similar to that of wild-type rt-PA, but its binding to fibrin is five times lower [14].

Duteplase is an analogue of the rt-PA double chain and has been studied in myocardial infarction [14].

Tenecteplase (TNK-rt-PA) is a mutant variant of rt-PA in which the removal of the glycosylation site results in a longer half-life and increases resistance to PAI-1 while retaining fibrin-binding properties [14].

Lanoteplase (n-PA) is a deletion and the only variant of the point mutation Asn117 $\rightarrow$ Gln rt-PA, devoid of the finger region and the epidermal growth factor domain, with an increased plasma half-life compared to alteplase [14].

Monteplase is a TPA mutant that has a long half-life. Monteplase is a TPA mutant with a long half-life [14].

Pamiteplase is a TPA mutant with a longer half-life and improved fibrin binding compared to analogs [14].

Streptokinase is a $47 \mathrm{kDa}$ protein produced by various strains of $\beta$-hemolytic streptococci. Unlike UPA and TPA, which themselves have proteolytic activity, streptokinase is not an enzyme, but acquires the ability to indirectly activate human plasminogen, forming a 1: 1 complex with plasminogen or plasmin, in which the catalytic site of cymogen is activated non-proteolytically by intramolecular cleavage of the Arg560Val561 bond. The resulting activator complex is a highly specific enzyme that binds free plasminogen and converts it to plasmin by proteolytic cleavage. In addition, plasmin cleaves the streptokinase molecule, generating the N-terminal 59-terminal fragment, which remains covalently attached to the rest of the molecule, preventing the streptokinaseplasminogen complex from binding to fibrin [9]. The advantage of streptokinase is its low cost in production, however, this protein can cause immunological complications and lead to generalized proteolysis with concomitant bleeding [13, 14]

Anistreplase or acylated Plg-SK activator complex is a streptokinase derivative that has a longer plasma half-life [14].

The active form of UPA (tcu-PA), currently produced recombinantly, is used as a thrombolytic agent, as well as recombinant pro-yurokinase or scu-PA (saruplase) [14].

Desmoteplase is a plasminogen activator derived from the saliva of the vampire bat Desmodus rotundus, which lacks a second kringle site and a plasmin-sensitive t-PA cleavage site and is highly fibrin dependent and fibrin specific [14].

Plasmin and microplasmin in their truncated form are considered direct thrombolytic substances [14].

Staphylokinase is a $15.5 \mathrm{kDa}$ protein produced by Staphylococcus aureus, which does not possess enzymatic activity, but forms a 1: 1 complex with trace amounts of plasmin on fibrin, which activates other plasminogen molecules [10]. In the blood plasma, in the absence of fibrin, the trace elements of free plasmin and the activity of the plasminogen activator in the staphylokinase-plasmin complex are rapidly inhibited by the $\alpha 2$-antiplasmin protein, but in the presence of fibrin, the generation of the active staphylokinase-plasmin complex is facilitated by the fact that the initial microelements of fibrin-bound plasmin are protected from $\alpha 2$-antiplasmin and inhibition complex on the surface of the clot is significantly delayed, and the activation of plasminogen is enhanced $[13,14]$.

It has been shown that recombinant staphylokinase is more specific for fibrin than rtPA in patients with progressive myocardial infarction, but can elicit an immune response. [14]. 
A promising aspect of thromboprophylaxis and therapeutic thrombolysis is the use of substances that stimulate the release of endogenous t-PA, such as a vasopressin (desmopressin) derivative, some acylated dipeptides and a pyrimidine derivative [14].

\subsection{Use of proteases from fungi and bacteria as thrombolytic agents}

Over time, scientists have successfully discovered the widespread use of proteases in medicine. In medicine, various formulas such as gauze, nonwoven fabrics, and ointment containing alkaline proteases produced by B. subtilis have promising therapeutic properties. Certain lytic enzyme deficiency syndromes are diagnosed by oral administration of alkaline proteases. Fibrin degradation has been reported to have been achieved by alkaline fibrinolytic proteases. The use of this fibrinolytic enzyme suggests its further use as an anticancer drug and in thrombolytic therapy. In therapeutic use, a slow-release dosage form preparation containing collagenases with alkaline proteases is widely used. When collagen is hydrolyzed by the enzyme, low molecular weight peptides are released without the release of amino acids for therapeutic use. For the treatment of various diseases, such as burns, carbuncles, boils and wounds, an elastoterase preparation immobilized on a bandage is used [15].

One of the poorly studied sources of thrombolytic substances is xylotrophic basidiomycetes. Producers of fungal thrombolytic enzymes, as well as the thrombolytic drugs themselves, which have a direct medical purpose, in Russia were initially studied and characterized in detail in various groups of micromycetes. As an example, we can point to the fibrinolytic preparation "terrilitin", developed on the basis of the use of the soil micromycete Aspergillus terricola and proposed for practical implementation. However, a narrow range of microbial thrombolytic drugs and the presence of serious side effects in them (primarily due to the high level of general, non-specific proteolysis) still restrain their widespread use in medical practice. The biosynthesis of peptide hydrolases of fibrin- and thrombolytic action in basidiomycetes remained practically terra incognita until the end of the twentieth century. The lack of sufficiently complete information on the level of fibrinolytic and thrombolytic activities in different representatives of macromycetes and the deficiency of these enzymes in medical practice have determined the relevance of this line of research. Unfortunately, the search and study of new producers of thrombolytic enzymes today continue to be an urgent problem of practical medicine in many countries of the world. The first targeted studies of fibrinolytic (FA) and thrombolytic activities (TA) of basidial macromycetes were started in Russia in the laboratory of fungal biochemistry at the V.I. V.L. Komarov of the Russian Academy of Sciences. One of the first in this direction were the works of N.N. Falina on the study of cultures Flammulina velutipes (Curt.:Fr.) P. Karst. [3]. Later, these studies were continued on a wide mycological material, including cultures and fruiting bodies of basidiomycetes from various taxonomic and ecological groups [16].

\section{Conclusions}

The variety of fibrinolytic agents and their factors and cofactors influencing the process of dissolution of a blood clot together constitute a difficult task for the researcher. When, as in emergency treatment, a quick and safe fibrinolytic agent is needed, in case of a deviation in the functioning of the fibrinolytic system, a thorough analysis of the causes of the disease is necessary. One of the interesting and promising tasks in this area is the production of fibrinolytic and thrombolytic enzymes from the culture liquid of basidiomycetes. 


\section{References}

1. D.T. Jamison, H. Gelband, S. Horton, Disease Control Priorities: Improving Health and Reducing Poverty. 3rd edition (Washington (DC): The International Bank for Reconstruction and Development (The World Bank, 2017) DOI: 10.1596/978-1-46480527-1_ch4

2. World Health Organization, The top 10 causes of death (Geneva: World Health Organization, 2018) https://www.who.int/news-room/fact-sheets/detail/the-top-10causes-of-death

3. E.V. Konstantinova, N.A. Shostak, M.Y. Gilyarov, The Clinician 9(1), 4-12 (2015) DOI: 10.17650/1818-8338-2015-1-4-12

4. V.V. Krylov, S.A. Burov, V.G. Dash'yan, I.E. Galankina, Annals of the Russian academy of medical sciences 68(7), 24-31 (2013) DOI: 10.15690/vramn.v68i7.708

5. P. Thornton, J. Douglas, Best practice \& research Clinical obstetrics \& gynaecology 24(3), 339-352 (2010) DOI: 10.1016/j.bpobgyn.2009.11.010

6. A.S. Zhalyalov, A.N. Balandina, A.D. Kuprash, A. Shrivastava, A.M. Shibeko, Pediatric Hematology/Oncology and Immunopathology 16(1), 69-82 (2017) DOI: 10.24287/ 1726-1708-2017-16-1-69-82

7. M. Pathak, B.G. Kaira, A. Slater, J. Emsley, Cell receptor and cofactor interactions of the contact activation system and factor XI, Frontiers in Medicine 5, 66 (2018) DOI: 10.3389/fmed.2018.00066

8. J. Konings, L. Hoving, R.S. Ariëns, E.L. Hethershaw, M. Ninivaggi, L.J. Hardy, B. de Laat, H. ten Cate, H. Philippou, J.W. Govers-Riemslag, Thrombosis research 136(2), 474-480 (2015) DOI: 10.1016/j.thromres.2015.06.028

9. J.J. Sidelmann, J. Gram, J. Jespersen, C. Kluft, Seminars in Thrombosis and Hemostasis 26(6), 605-618 (2000) DOI: 10.1055/s-2000-13216

10. P.J. Gaffney, Molecular pathology of the multiple forms of fibrinogen and its fragments. (Isozymes V2: Physiological Function, 1975) DOI: 10.1016/B978-0-12472702-1.50027-X

11. S.M. Bates, Seminars in thrombosis and hemostasis 38(7), 673-682 (2012) DOI: $10.1055 / \mathrm{s}-0032-1326782$

12. A. Noori, S.J. Ashrafi, R. Vaez-Ghaemi, A. Hatamian-Zaremi, T.J. Webster, International journal of nanomedicine 12, 4937 (2017) DOI: 10.2147/IJN.S124671

13. R.I. Litvinov, Kazan medical journal 94(5), $711-718$ (2013) DOI: 10.17816/KMJ1926

14. H.I. Saba, H.R. Roberts, Hemostasis and thrombosis (Chichester: John Wiley \& Sons, 2014) DOI: 10.1002/9781118833391

15. A. Razzaq, S. Shamsi, A. Ali, Q. Ali, M. Sajjad, A. Malik, M. Ashraf, Frontiers in bioengineering and biotechnology 7, 110 (2019) DOI: 10.3389/fbioe.2019.00110

16. N.P. Denisova, International Journal of Medicinal Mushrooms 12(3), 317-325 (2010) DOI: 10.1615/IntJMedMushr.v12.i3.110 